CONCISE REPORT

\title{
Differential regulation of the bone morphogenic protein antagonist chordin in human normal and osteoarthritic chondrocytes
}

\author{
G Tardif, J-P Pelletier, D Hum, C Boileau, N Duval, J Martel-Pelletier
}

Ann Rheum Dis 2006;65:261-264. doi: 10.1136/ard.2005.037523

Objective: To investigate the distribution of the bone morphogenic protein (BMP) antagonist chordin in normal and osteoarthritic cartilage and synovial membranes, and its regulation in chondrocytes and synovial fibroblasts by inflammatory and growth factors.

Methods: Localisation of chordin in tissues was undertaken by immunohistochemistry and gene regulation was determined by real time polymerase chain reaction.

Results: In normal cartilage, chordin was found at low levels (mean (SD), $7.6(1.3) \%$ ), mainly in the very superficial layers. In osteoarthritis, chordin was also found in the superficial layers $(8.9(1.1) \%)$, though at a significantly higher level $(24.7(1.5) \%)$ in the last two thirds of the cartilage. In contrast to normal cells, chordin mRNA and protein levels were significantly downregulated $(p<0.01)$ in osteoarthritic chondrocytes by all the growth factors tested. Interferon $\gamma$ stimulated chordin expression in normal but not in osteoarthritic chondrocytes $(p<0.0002)$, while interleukin $1 \beta$ and tumour necrosis factor $\alpha$ did not affect the expression level. However, no difference was found in either the distribution or regulation of chordin in normal and osteoarthritic synovial membranes or synovial fibroblasts.

Conclusions: The differential distribution and regulation of chordin in normal and osteoarthritic cartilage and chondrocytes suggests an involvement of this antagonist in the osteoarthritic process.

B one morphogenic proteins (BMPs) are secreted factors that play essential roles during embryonic development and adult processes such as bone remodelling and repair, cartilage maintenance, and wound healing. ${ }^{1-4}$ BMP activities are modulated by several unrelated secreted proteins collectively known as BMP antagonists, which bind to the BMPs and prevent their binding and signalling through their specific receptors.[3 4] Each antagonist differs in its specificity and affinity to the different BMPs. ${ }^{5-8}$

Chordin binds BMP-2, BMP-4, and BMP-7, though with the highest affinity to BMP-2 and BMP-4. We recently reported that chordin was expressed in human articular chondrocytes and synovial fibroblasts'; however, information about its regulation in these cells is lacking. The aim of the present study was to localise chordin in normal and osteoarthritic articular cartilage and synovial membrane, and to investigate its regulation in normal and osteoarthritic chondrocytes and synovial fibroblasts following treatment by inflammatory and growth factors. The data showed a differential distribution and regulation of chordin in normal and osteoarthritic cartilage/chondrocytes, but not in synovial membranes or synovial fibroblasts.

\begin{abstract}
METHODS
Factors

Recombinant human interleukin $1 \beta$ (ILl $\beta$ ) was obtained from Genzyme (Cambridge, Massachusetts, USA), activin A (Act.A), basic fibroblast growth factor (bFGF), epithelial growth factor (EGF), interferon $\gamma$ (IFN $\gamma$ ), platelet derived growth factor $\mathrm{BB}$ (PDGF-BB), transforming growth factor $\beta 1$ (TGF $\beta 1$ ), tumour necrosis factor $\alpha$ (TNF $\alpha)$, and BMP-2 were obtained from R\&D Systems (Minneapolis, Minnesota, USA). Each factor was used at $10 \mathrm{ng} / \mathrm{ml}$ except for IFN $\gamma(10 \mathrm{U} / \mathrm{ml})$, $\operatorname{ILl} \beta(100 \mathrm{pg} / \mathrm{ml})$, and $\mathrm{TNF} \alpha(5 \mathrm{ng} / \mathrm{ml})$.
\end{abstract}

\section{Specimen selection}

Human normal cartilage (femoral condyles, tibial plateaus) and synovial membranes were obtained from individuals within 12 hours of death (mean (SEM) age, 66 (5) years) who had no history of joint disease and died of causes unrelated to arthritic diseases. Human osteoarthritic cartilage and synovial membranes were obtained from patients undergoing total knee arthroplasty (age 71 (9) years) and represented moderate to severe osteoarthritis as defined according to macroscopic criteria. At the time of surgery, patients were treated with acetaminophen, non-steroidal anti-inflammatory drugs, or selective cyclooxygenase-2 inhibitors. None had received intra-articular steroid injections within three months before surgery.

The ethics committee board of the Hôpital Notre-Dame approved the use of the human articular tissues.

\section{Cellular and explant cultures}

Chondrocytes and synovial fibroblasts were released from full thickness strips of whole articular cartilage and synovial membrane, respectively, and cultured as previously described. ${ }^{9}{ }^{10}$ Cultured chondrocytes were used at first passage and synovial fibroblasts at second or third passage.

The effects of factors on gene transcription were assessed by incubating confluent cells for 24 hours in Dulbecco's modified Eagle's medium (DMEM; Gibco-BRL, Burlington, Ontario, Canada) $/ 0.5 \%$ fetal calf serum (FCS; Invitrogen Canada, Burlington, Ontario) containing the factors under study.

Osteoarthritic cartilage explants $(\sim 150 \mathrm{mg})$ were incubated for 72 hours in DMEM/2.5\% FCS with the factors under study, after which they were processed for immunohistochemistry.

Abbreviations: Act.A, activin A; BMP, bone morphogenic protein; bFGF, basic fibroblast growth factor; EGF, epithelial growth factor; IFN $\gamma$, interferon $\gamma ;$ IL1 $\beta$, interleukin $1 \beta$; PDGF-BB, platelet derived growth factor $\mathrm{BB} ; \mathrm{TGF} \beta 1$, transforming growth factor $\beta 1 ; \mathrm{TNF} \alpha$, tumour necrosis factor $\alpha$ 

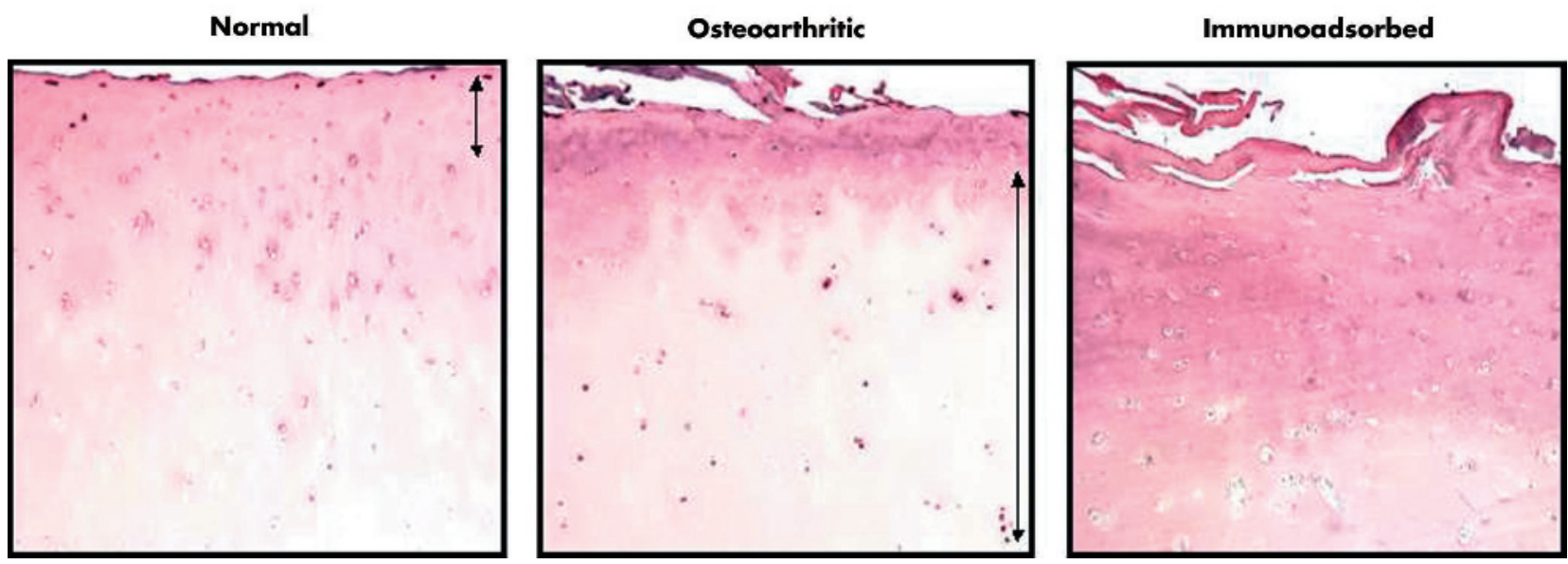

Figure 1 Chordin immunostaining in representative sections of human normal and osteoarthritic cartilage. Chordin is located preferentially at the superficial zone of normal cartilage, and at the superficial and intermediate zones of osteoarthritic cartilage. No specific staining was detected in the osteoarthritic cartilage immunoadsorbed with a 20 -fold molar excess of the peptide. Arrows indicate the superficial and intermediate zones. (Original magnification $\times 60$.)

\section{RNA extraction, reverse transcription and real time polymerase chain reaction}

RNA extraction, reverse transcription, and real time polymerase chain reaction (PCR) were done as described previously. ${ }^{9}$ Primer sequences were 5'-CGCATCAGTGGAC ACATTG (sense) and 5'-TTCTGCAGCAGCATATGAGC (antisense) for chordin, 5'-CAGAACATCATCCCTGCCTCT (sense) and 5'-GCTTGACAAAGTGGTCGTTGAG (antisense) for GAPDH. The data were processed with GeneAmp 5700 SDS software and given as threshold cycle $\left(\mathrm{C}_{\mathrm{T}}\right)$. Change in gene expression was calculated as -fold change $=2^{-\Delta(\Delta \mathrm{CT})}$, where $\Delta \mathrm{C}_{\mathrm{T}}=\mathrm{C}_{\mathrm{T} \text { stimulated }}-\mathrm{C}_{\mathrm{T} \text { GAPDH }}$, and $\Delta(\Delta \mathrm{CT})=\Delta \mathrm{C}_{\mathrm{T} \text { stimulated }}-$ $\Delta \mathrm{C}_{\mathrm{T} \text { control. }}$ The primer efficiencies for chordin and GAPDH were similar.

\section{Synovial membrane histology}

Specimens were prepared and graded on a scale of 0 to 10 by two independent observers, as previously described. ${ }^{11}$

\section{Immunohistochemistry}

Cartilage specimens were processed for immunohistochemistry as previously described, ${ }^{9}$ and at comparable sites. The primary antibody was a goat polyclonal antihuman chordin (IgG fraction; Santa Cruz Biotechnology, Santa Cruz, California, USA), used at $4 \mu \mathrm{g} / \mathrm{ml}$. Staining specificity was determined by substituting the primary antibody with preimmune goat IgG (Chemicon, Temecula, California, USA) used at the same concentration as the primary antibody, and the use of a 20-fold molar excess of the peptide employed to produce the chordin antibody (Santa Cruz Biotechnology). Both controls showed only background staining.

The total numbers of chondrocytes and of positively stained chondrocytes were counted separately, from three fields of the superficial (upper third of the cartilage) and three fields of the intermediate (lower two thirds) zones. Results are expressed as percentage of positive chondrocytes. Each slide was evaluated by two observers with more than $95 \%$ agreement. The percentage of positive chondrocytes in cartilage explants was determined in the intermediate zone.

\section{Statistical analysis}

Data are expressed as mean (SEM). Statistical significance was assessed by the Student $t$ test, and $\mathrm{p}$ values $<0.05$ were considered significant.

\section{RESULTS \\ Production of chordin in cartilage and synovial membrane}

The data showed a differential distribution of chordin (fig 1). In normal cartilage $(n=6)$, chordin was produced at a low level mainly in the superficial zone (7.6 (1.3)\% positive cells). In osteoarthritis $(\mathrm{n}=6)$, chordin was found at a significantly higher level in the intermediate zone (24.7 (1.5)\%) than in the superficial zone $(8.9(1.1) \%)(\mathrm{p}<0.0001)$.

Synovial membrane histology showed a higher score for osteoarthritis $(\mathrm{n}=4)$ than for the normal samples $(\mathrm{n}=3)$, at $5.8(0.5) \vee 1.5(0.3)$, respectively $(\mathrm{p}<0.001)$. Chordin was detected only in the lining cells of both normal and osteoarthritic samples (data not shown).

\section{Regulation of chordin expression by inflammatory and growth factors}

To identify factors that may lead to the differential distribution of chordin, chondrocytes were treated with factors known to affect cartilage physiology and pathology. Chordin basal expression was calculated as the ratio of number of chordin molecules divided by the number of GAPDH molecules $(0.096(0.024)$ for normal $(n=5), 0.088(0.03)$ for osteoarthritis $(\mathrm{n}=5))$.

Neither ILI $\beta$ nor TNF $\alpha$ significantly affected chordin expression in chondrocytes (fig 2A). IFN $\gamma$ did not affect chordin expression in osteoarthritic chondrocytes but significantly stimulated (by 4.6-fold) its expression in normal chondrocytes. ILl $\beta$ had no effect on protein levels of osteoarthritic cartilage $(n=3-5)$, but a significant decrease occurred with TNF $\alpha$ and IFN $\gamma$ (fig $2 \mathrm{C}$ ).

All the growth factors tested reduced chordin expression and protein levels in osteoarthritic chondrocytes significantly (fig 2, panels B and C). Normal chondrocytes were not affected by growth factors, except for bFGF and PDGF-BB, which decreased chordin expression significantly, though not to the same extent as in osteoarthritic cells. The basal levels of chordin in synovial fibroblasts were $0.59(0.16) \times 10^{-3}$ for normal samples $(\mathrm{n}=6)$ and $0.45(0.08) \times 10^{-3}$ for osteoarthritis samples $(n=6)$. Normal and osteoarthritic cells responded similarly to each of the factors tested (table 1 ). ILI $\beta$ and TNF $\alpha$ had no significant effect, but IFN $\gamma$ caused a high degree of stimulation of chordin expression-by 6-fold and 5.4-fold, respectively-in normal and osteoarthritic 

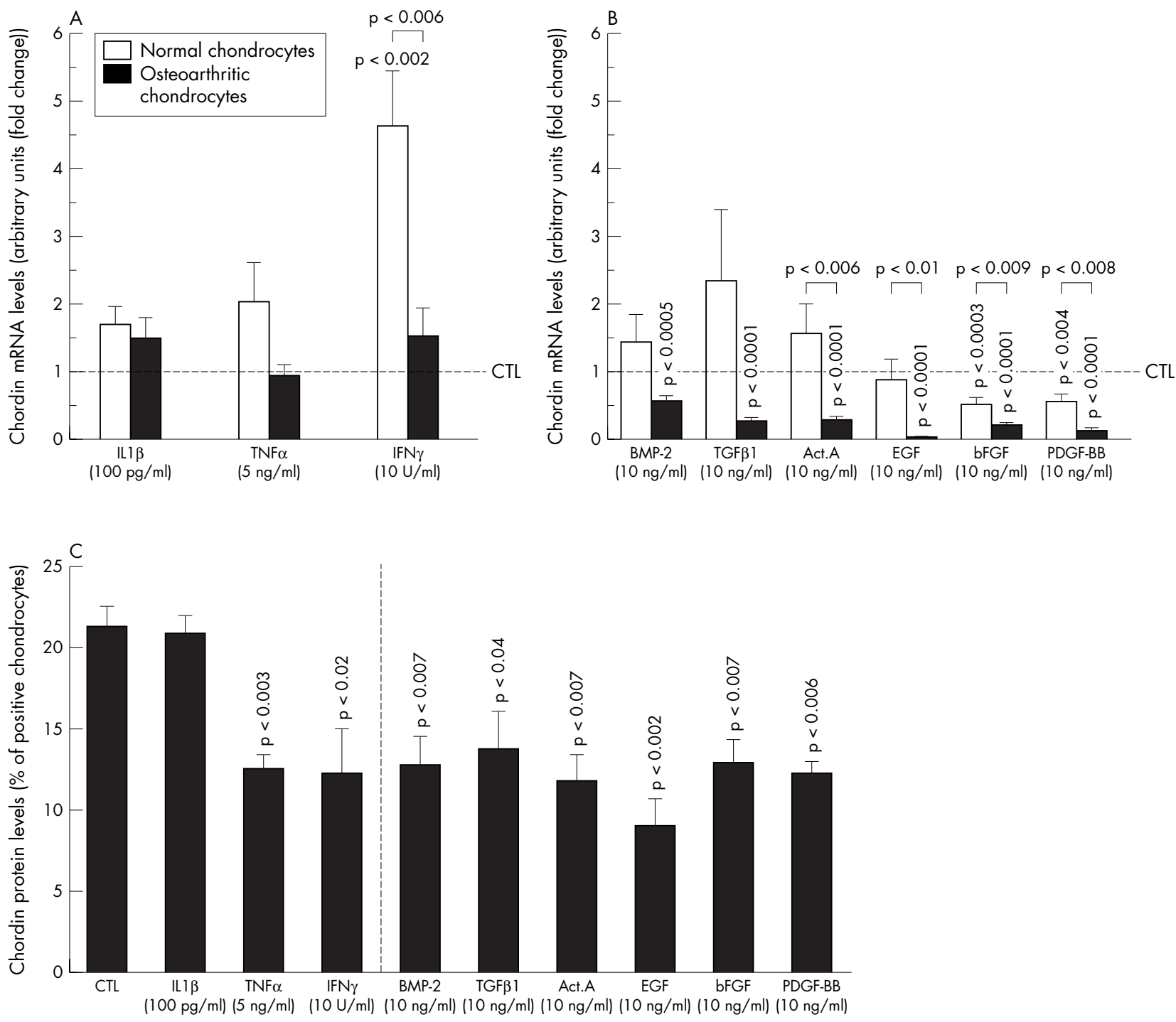

Figure 2 Chordin regulation by inflammatory and growth factors. Normal ( $n=5$, white bars) and osteoarthritic chondrocytes ( $n=5$, black bars) were incubated in the absence or presence of inflammatory factors (A) and growth factors (B). Total RNA was extracted and processed for real time polymerase chain reaction as described in Methods. Control (CTL) levels were given an arbitrary value of 1 , and the data are expressed as the mean of -fold change. (C) Protein levels in cultured osteoarthritic cartilage explants. Cartilage explants $(n=3-5)$ were incubated in the absence or presence of inflammatory and growth factors. Protein levels in the intermediate zone of the cartilage were determined by immunohistochemistry as described in Methods. Statistical significance was assessed by the Student $t$ test $v$ control. Error bars $=$ SEM in all panels. Act. A, activin A; BMP-2, bone morphogenic protein type 2; bFGF, basic fibroblast growth factor; EGF, epithelial growth factor; IFN $\gamma$, interferon $\gamma$; IL1 $\beta$, interleukin $1 \beta$; PDGF-BB, platelet derived growth factor BB; TGF $\beta 1$, transforming growth factor $\beta 1$; TNF $\alpha$, tumour necrosis factor $\alpha$.

synovial fibroblasts. bFGF and PDGF-BB reduced chordin expression significantly, but the other growth factors did not.

\section{DISCUSSION}

This study showed that chordin is differentially located and regulated in normal and osteoarthritic cartilage and chondrocytes, but not in synovial membrane or synovial fibroblasts, suggesting involvement of chordin in osteoarthritic cartilage. Although we previously reported that basal mRNA chordin levels were not significantly different between normal and osteoarthritic chondrocytes, ${ }^{9}$ the present study reveals that its protein distribution within the cartilage differs, with a significantly higher level being found in osteoarthritis than in normal cartilage.

The differential distribution in cartilage might reflect different regulation between normal and osteoarthritic chondrocytes. Although our data did not identify the factors responsible for the upregulation of chordin, there were some interesting findings. In contrast to normal chondrocytes, osteoarthritic cells did not increase chordin expression in response to IFN $\gamma$. This is not because of the lack of specific membrane receptors or their functionality, as IFN $\gamma$ has previously been shown to increase the production of another BMP antagonist, follistatin, in osteoarthritic chondrocytes. ${ }^{9}$ The IFN $\gamma$ signalling pathways leading to chordin expression might be altered in osteoarthritic chondrocytes. Furthermore, all growth factors tested downregulated chordin expression and production in osteoarthritis. The presence of chordin in the intermediate zone of the diseased tissue indicates that this BMP antagonist may primarily be involved in the remodelling process of this tissue.

A notable finding was that BMP-2, previously shown to stimulate the BMP antagonist gremlin significantly," decreased chordin levels in osteoarthritic chondrocytes. As the promoter regions of gremlin and chordin have not been cloned, nor their activities evaluated in vivo, the mechanisms 
Table 1 Effects of inflammatory and growth factors on chordin expression in normal and osteoarthritic synovial fibroblasts

\begin{tabular}{|c|c|c|c|c|}
\hline & $\begin{array}{l}\text { Normal } \\
\text { fibroblasts }\end{array}$ & $\mathrm{p}$ Value & $\begin{array}{l}\text { Osteoarthritic } \\
\text { fibroblasts }\end{array}$ & p Value \\
\hline \multicolumn{5}{|c|}{ Inflammatory factors } \\
\hline ILI $\beta$ & $1.09(0.18)$ & & $0.77(0.06)$ & \\
\hline TNF $\alpha$ & $1.01(0.25)$ & & $1.90(0.60)$ & \\
\hline $\mathrm{IFN} \gamma$ & $6.11(2.04)$ & $<0.02$ & $5.44(0.73)$ & $<0.0002$ \\
\hline \multicolumn{5}{|c|}{ Growth factors } \\
\hline BMP2 & $1.32(0.25)$ & & $1.43(0.37)$ & \\
\hline TGF $\beta 1$ & $1.19(0.24)$ & & $1.12(0.19)$ & \\
\hline Act.A & $1.97(0.89)$ & & $1.05(0.33)$ & \\
\hline EGF & $0.54(0.23)$ & & $0.66(0.19)$ & \\
\hline bFGF & $0.49(0.11)$ & $<0.0007$ & $0.53(0.13)$ & $<0.003$ \\
\hline PDGF-BB & $0.25(0.08)$ & $<0.0001$ & $0.27(0.10)$ & $<0.0001$ \\
\hline
\end{tabular}

Normal $(n=6)$ and osteoarthritic $(n=6)$ synovial fibroblasts were incubated in the absence (control) or presence of the factors. Concentrations for the inflammatory factors were IL $1 \beta, 100 \mathrm{pg} / \mathrm{ml} ; \mathrm{TNF} \alpha, 5 \mathrm{ng} / \mathrm{ml} ; \mathrm{IFN} \gamma, 10 \mathrm{U} / \mathrm{ml}$, and $10 \mathrm{ng} / \mathrm{ml}$ for all the growth factors. Control levels were given an arbitrary value of 1 , and values are the mean of -fold change (SEM). Statistical analysis was assessed by Student $t$ test $v$ control.

Act.A, activin A; BMP, bone morphogenic protein; bFGF, basic fibroblast growth factor; EGF, epithelial growth factor; IFN $\gamma$, interferon $\gamma ;$ IL1 $\beta$, interleukin 1 $\beta$; PDGF-BB, platelet derived growth factor BB; TGF $\beta 1$, transforming growth factor $\beta 1 ; \mathrm{TNF} \alpha$, tumour necrosis factor $\alpha$.

of BMP-2 actions on each promoter are yet to be elucidated. BMP-2 is upregulated in osteoarthritic articular tissues ${ }^{12}{ }^{13}$ and is produced mostly in the middle and deep zones of osteoarthritic cartilage, ${ }^{12}$ where gremlin ${ }^{9}$ and chordin are located. The higher levels of BMP-2 could explain increased levels of gremlin in the pathological cartilage, but factors responsible for chordin upregulation remain unknown. Chordin regulation is likely to be complex, as the gene encodes several alternative spliced variants with opposing BMP activities. ${ }^{14} \mathrm{~A}$ thorough investigation of these variants might provide more information about chordin regulation. Because of its BMP binding activity, its regulation by growth factors but not by inflammatory factors, its location, and its increased levels, chordin probably plays a role in the remodelling and repair of osteoarthritic cartilage rather than in the inflammatory processes.

Winkler et $a^{15}$ recently reported that the BMP antagonists noggin and sclerostin could bind to each other with high affinity and mutually attenuate their activity. Because gremlin and chordin are at similar locations in osteoarthritic cartilage and bind to the same BMPs, a possible interaction between them is an interesting speculation, though as yet unconfirmed. It could be hypothesised that chordin has a dual role in cartilage remodelling: reducing BMP activity by its direct binding to BMPs, and permitting BMP activity by its potential binding to gremlin.

\section{ACKNOWLEDGEMENTS}

We thank Martin Boily for his expert technical assistance in immunohistochemistry, and Santa Fiori and Virginia Wallis for the manuscript preparation.

\section{Authors' affiliations}

G Tardif, J-P Pelletier, D Hum, C Boileau, J Martel-Pelletier, Osteoarthritis Research Unit, Hôpital Notre-Dame, Quebec, Canada N Duval, Pavillon des Charmilles, Vimont, Quebec

Correspondence to: Dr Johanne Martel-Pelletier, Osteoarthritis Research Unit, Hôpital Notre-Dame, Centre Hospitalier de l'Université de Montréal, 1560 Sherbrooke Street East, Montreal, Quebec, Canada H2L 4M1; jm@martelpelletier.ca

\section{Accepted 11 July 2005}

\section{REFERENCES}

1 Ducy $P$, Karsenty $G$. The family of bone morphogenetic proteins. Kidney Int 2000;57:2207-14.

2 Edwards CJ, Francis-West PH. Bone morphogenetic proteins in the development and healing of synovial joints. Semin Arthritis Rheum 2001;31:33-42.

3 Balemans W, Van Hul W. Extracellular regulation of BMP signaling in vertebrates: a cocktail of modulators. Dev Biol 2002;250:231-50.

4 Canalis E, Economides AN, Gazzerro E. Bone morphogenetic proteins, their antagonists, and the skeleton. Endocr Rev 2003;24:218-35.

5 Piccolo S, Sasai Y, Lu B, De Robertis EM. Dorsoventral patterning in Xenopus: inhibition of ventral signals by direct binding of chordin to BMP-4. Cell 1996;86:589-98.

6 Zimmerman LB, De Jesus-Escobar JM, Harland RM. The Spemann organizer signal noggin binds and inactivates bone morphogenetic protein 4. Cell 1996;86:599-606

7 Hsu DR, Economides AN, Wang X, Eimon PM, Harland RM. The Xenopus dorsalizing factor Gremlin identifies a novel family of secreted proteins that antagonize BMP activities. Mol Cell 1998;1:673-83.

8 lemura S, Yamamoto TS, Takagi C, Uchiyama H, Natsume T, Shimasaki S, ef al. Direct binding of follistatin to a complex of bone-morphogenetic protein and its receptor inhibits ventral and epidermal cell fates in early Xenopus embryo. Proc Natl Acad Sci USA 1998;95:9337-42.

9 Tardif G, Hum D, Pelletier JP, Boileau C, Ranger P, Martel-Pelletier J. Differential gene expression and regulation of the bone morphogenetic protein antagonists follistatin and gremlin in normal and osteoarthritic human chondrocytes and synovial fibroblasts. Arthritis Rheum 2004;50:2521-30.

10 Reboul P, Pelletier JP, Tardif G, Cloutier JM, Martel-Pelletier J. The new collagenase, collagenase- 3 , is expressed and synthesized by human chondrocytes but not by synoviocytes: a role in osteoarthritis. J Clin Invest 1996;97:2011-19.

11 Pelletier JP, Martel-Pelletier J, Ghandur-Mnaymneh L, Howell DS, Woessner JF. Role of synovial membrane inflammation in cartilage matrix breakdown in the Pond-Nuki dog model of osteoarthritis. Arthritis Rheum 1985;28:554-61.

12 Fukui N, Zhu Y, Maloney WJ, Clohisy J, Sandell U. Stimulation of BMP-2 expression by pro-inflammatory cytokines IL-1 and TNF-alpha in normal and osteoarthritic chondrocytes. J Bone Joint Surg Am 2003;85-A(suppl 3):59-66.

13 Nakase T, Miyaji T, Tomita T, Kaneko M, Kuriyama K, Myoui A, et al. Localization of bone morphogenetic protein- 2 in human osteoarthritic cartilage and osteophyte. Osteoarthritis Cartilage 2003;1 1:278-84.

14 Millet C, Lemaire P, Orsetti B, Guglielmi P, Francois V. The human chordin gene encodes several differentially expressed spliced variants with distinct BMP opposing activities. Mech Dev 2001;106:85-96.

15 Winkler DG, Yu C, Geoghegan JC, Ojala EW, Skonier JE, Shpektor D, et al. Noggin and sclerostin bone morphogenetic protein antagonists form a mutually inhibitory complex. J Biol Chem 2004;279:36293-8. 